\title{
Analysis of the Vertical Stiffness of Rolling Guide that Involves the Elastic Deformation of Carriage Skirt
}

\author{
Li Jinfeng*, Wang Liping and Guan Liwen \\ Department of Mechanical Engineering, Tsinghua University, Beijing, 100084, P.R. China
}

\begin{abstract}
Static stiffness is an important indicator of the performance of a rolling guide, having direct influence on the stiffness and precision of computer numerically controlled (CNC) machine tools. After preloading the rolling guide, an outward elastic deformation is generated at the carriage skirt, which leads to a decrease in the static stiffness of the rolling guide. Therefore, there would be relatively large errors between the numerical results and the experimental results when the carriage is considered as a rigid body. In this paper, an analytical method for estimating the vertical stiffness of rolling guide was proposed, which took into account the elastic deformation in the carriage skirt. The contact elastic deformation model under loads was given using Hertz's contact theory, from which the numerical results for the vertical stiffness of the surface of the rolling guide was calculated when the elastic deformation in the carriage skirt was ignored. The calculation method for the carriage skirt deformation was given using the finite element method, from which the numerical relationship between the deformation and the contact force was obtained after fitting adjustment. An analytical model was therefore established and took into account the elastic contact deformation and the carriage skirt deformation, and a universal calculation method was proposed for vertical stiffness. Experimental results show that compared to those not involving the deformation, the numerical results for vertical stiffness involving the carriage skirt deformation matched more closely with the experimental results, with relative errors no greater than $6.5 \%$.
\end{abstract}

Keywords: Carriage skirt, rolling guide, vertical stiffness.

\section{INTRODUCTION}

With the wide application of roller guides in all kinds of high-speed and high precision equipment, the static stiffness of roller guide has received more attention than ever in the academic world. Researchers around the world have conducted a series of studies on the accurate measurement of the static stiffness of roller guide since the 1990s.

Sun et al. [1,2] were the first to calculate the static stiffness of a vertically preloaded linear rolling guide using Hertz's contact theory. Shimizu [3] also analyzed the static stiffness of the linear rolling guide system. These studies were all conducted under the assumption that both the carriage and groove were rigid bodies, and only the elastic contact between the groove and rolling ball was taken into account, while the influence of carriage or guide deformation on vertical stiffness was ignored, resulting in a much smaller measured stiffness than the calculated stiffness. Therefore, scholars have turned to study the carriage skirt deformation, considering it an important factor in reducing the vertical static stiffness.

The configuration of a carriage is usually irregular, and it is difficult to assess its skirt deformation by calculation or direct measurement, which makes it difficult to calculate the corresponding vertical stiffness. Liu et al. [4] simplified the carriage into approximation mechanical models of two linear

*Address correspondence to this author at the Department of Mechanical Engineering, Tsinghua University, Beijing, 100084, P.R. China;

Tel: 18800089398; E-mail: lijf08@mails.tsinghua.edu.cn angular springs, and calculated the rotational elasticity coefficient of those two angular springs using the finite element software, which eventually derived the stiffness of the carriage. Chen et al. [5] built a rolling guide vertical stiffness model that took into account the carriage skirt deformation based on the Hertz's contact theory and the elastic beam theory. In order to overcome the problems of the traditional rigid model, Ohta [6] built a flexible model that incorporates the flexibility of the carriage and the groove, and calculated the carriage and the groove flexible deformations using finite element analysis, which resulted in relative errors of 9 to $21 \%$ between the calculated stiffness and the measured stiffness.

Since the sizes of applied force vary, the contact area and direction of the contact force between the rolling ball and groove is not fixed. Therefore, iterative calculation was used based on the Hertz's theory and the finite element method [6]. However, when the number of iterations is very large, the calculation process is very complex since the boundary condition needs to be modified at each iteration, and that it is difficult to select the initial value.

Therefore, in this paper, a convenient and accurate calculation method for the vertical stiffness of a rolling guide was proposed. A force model for the carriage was built in the finite element software that calculated the deformations of the carriage under different preload stresses, fitting the preload stress with deformation. Also, a mathematical model between the rolling ball and rolling groove was built based on a combination of the Hertz's contact theory and the fitting equation. In addition, experiments were conducted on the 
vertical stiffness of the rolling guide, which verified the accuracy of the model built. Furthermore, the variation of deformation was analyzed after comparing the changes in carriage skirt deformation with the changes in preload stresses.

\section{THE CONTACT MODEL UNDER ELASTIC DEFORMATION}

\subsection{The Distance Between Groove Curvature Centers}

The distance between the curvature center at carriage groove and center at guide groove is the key to solving the contact force and contact angle in the contact theory. When an external force $F_{V}$ is applied, the changes of distance between groove curvature centers, contact force and contact angle are shown in Fig. (1). $d_{0}$ is defined as the diameter of the rolling ball, $\alpha_{0}$ is the initial contact angle between the rolling ball and the groove, $O_{c i}$ and $O_{r i}$ refer to the $\mathrm{i}^{\text {th }}$ curvature center of the carriage groove and guide groove, respectively. $r_{c}$ and $r_{r}$ refer to the curvature radius of the carriage groove and the guide groove, respectively. The theoretical distance $s_{0}$ between $O_{c i}$ and $O_{r i}$ is:

$s_{0}=r_{r}+r_{c}-d_{0}$

In order to increase the stiffness of the rolling guide and eliminate the gap, internal load is needed to apply to the rotating body in advance, which is called preload. Preloaded guide is usually needed for occasions that need high stiffness, high precision, and large loads. Interference fit is generated between preloaded rolling ball and the groove, which is defined as $\delta_{0}$ :

$\delta_{0}=d-d_{0}$

Fig. (1). Illustration of the forces applied to the guide rail.

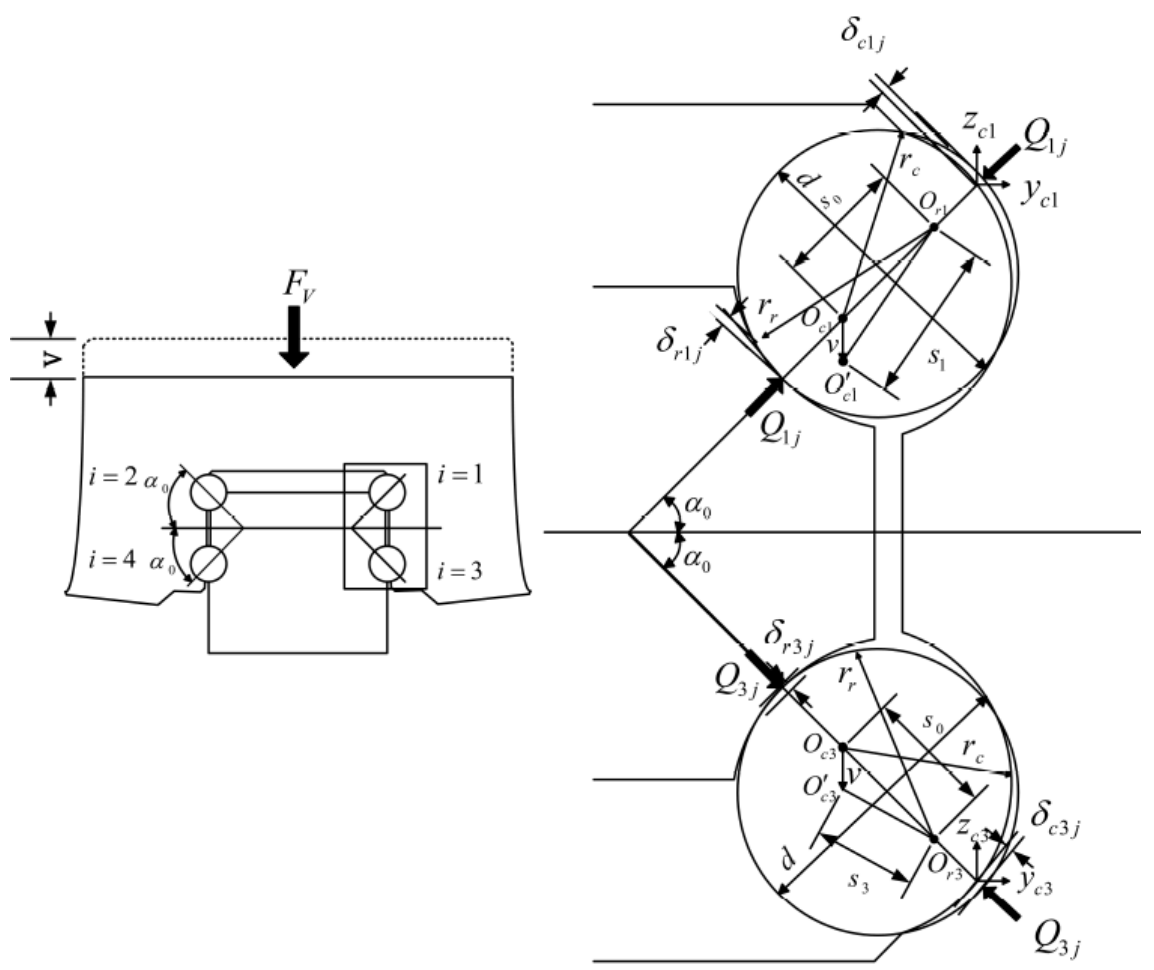

In which $\mathrm{d}$ refers to the diameter of the preloaded rolling ball. When Equation (2) is substituted into Equation (1):

$s_{0}=r_{r}+r_{c}-d_{0}+\delta_{0}=m_{0}+\delta_{0}$

In which $m_{0}=r_{r}+r_{c}-d$. The contact force generated at the $i^{\text {th }}$ groove and the $j^{\text {th }}$ rolling ball on the groove is marked as $Q_{i j}$, and the Hertz contact deformation generated between the $\mathrm{j}^{\text {th }}$ rolling ball at the $\mathrm{i}^{\text {th }}$ groove and the carriage groove is marked as $\delta_{c i j}$, and the Hertz contact deformation generated between this rolling ball and the guide groove is marked as $\delta_{r i j}$.

The carriage skirt has a tendency to expand outwardly under the vertical force; therefore not only Hertz contact deformation would be generated at the carriage groove, but also the elastic deformation would be generated under the contact force. The deformation at the $y$ direction of the $i^{\text {th }}$ carriage groove is marked as $y_{c i}$, and that at the $\mathrm{z}$ direction is marked as $z_{c i}$. When an external force $F_{V}$ is applied as shown in Fig. (2), the curvature center of the carriage groove would change, which is marked as $O_{c i}^{\prime}$. $\alpha_{i}$ is the contact angle between the rolling ball and the $\mathrm{i}^{\text {th }}$ groove. $S_{i}$ is defined as the distance between the changed curvature center $O_{c i}{ }_{c i}$ and $O_{r i}$. According to Fig. (2).

$s_{i}=\sqrt{\left(s_{0} \cos \alpha_{0}-y_{c i}\right)^{2}+\left(s_{0} \sin \alpha_{0}+z_{c i} \mp v\right)^{2}}$

where $\mathrm{v}$ refers to the deflection of the carriage in the direction of load. The plus and minus marks are relevant to the positions of the groove - the plus mark represents the upper groove, and the minus mark represents the bottom groove. The contact angle $\alpha_{i}$ can be obtained from Equation 
(5) as follows:

$\cos \alpha_{i}=\frac{s_{0} \cos \alpha_{0}-y_{c i}}{s_{i}}$

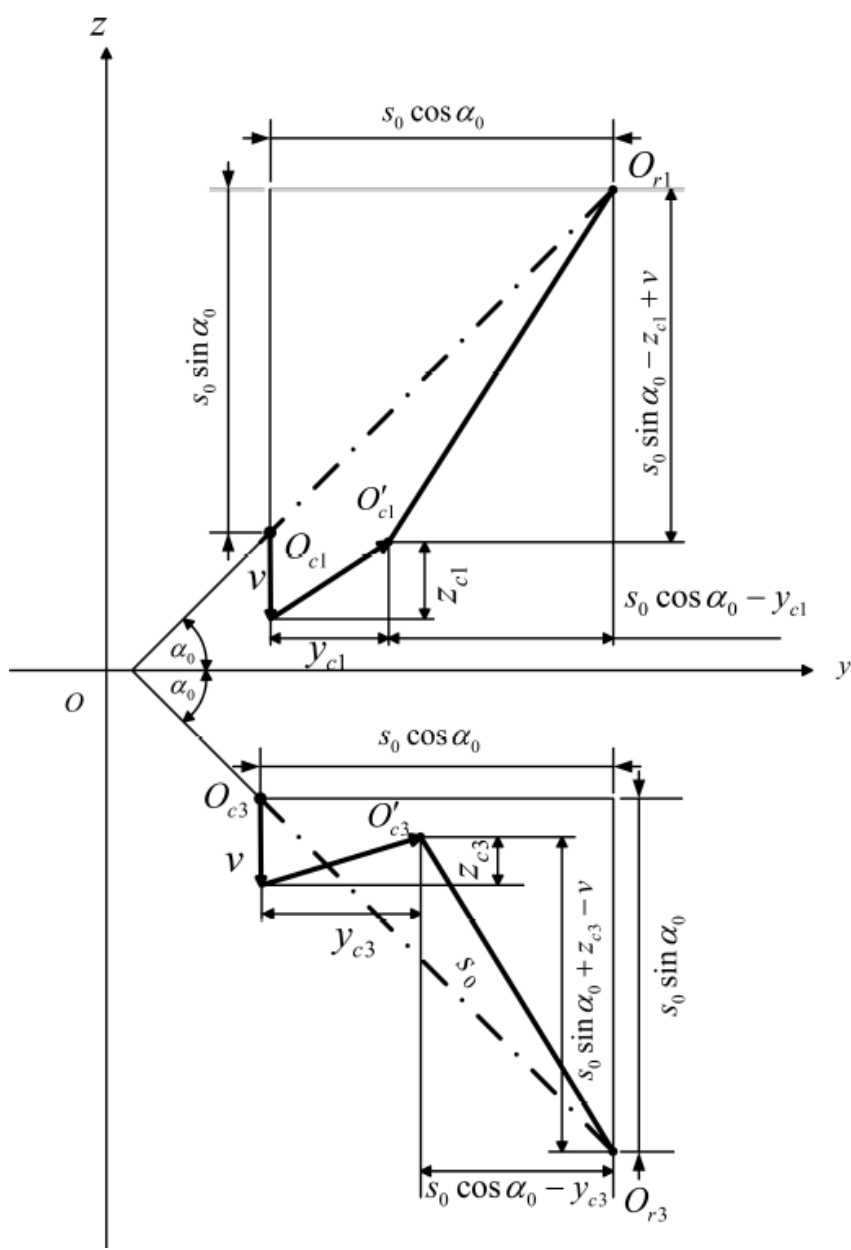

Fig. (2). Change in the position of the curvature center under a loaded state.

\subsection{Deformation Under the Hertz Contact}

There are several presumptions when using Hertz's contact theory to conduct the calculation: (1) the deformation generated at the contact area can be ignored compared to the radius of the rolling ball; (2) the influence of the manufacturing error and roughness of the groove and rolling ball on the load distribution of the contact force could be ignored; (3) the change in contact angle resulted from the deformation generated in the contact area can be ignored. The Hertz deformation $\delta_{i j}$ is composed of the deformation $\delta_{c i j}$ generated from the carriage groove and rolling ball, and the deformation $\delta_{r i j}$ generated from the guide groove and rolling ball, as shown in Equation (6):

$\delta_{i j}=\delta_{c i j}+\delta_{r i j}=s_{i}-m_{0}$

According to the Hertz's theory, the deformation $\delta$ generated at the point contact between the rolling ball and the groove is: $\delta=\frac{2 K}{\pi \mu} \sqrt[3]{\frac{1}{8}\left[\frac{3}{2}\left(\frac{1-v_{1}^{2}}{E_{1}}+\frac{1-v_{2}^{2}}{E_{2}}\right)\right]^{2} \sum \rho} \cdot Q^{2 / 3}$

where $Q$ refers to the normal pressure applied on the rolling ball, and $E_{l}$ and $v_{l}$ refer to the elastic modulus of the rolling ball material and the Poisson's ratio. $E_{2}$ and $v_{2}$ refer to the elastic modulus of the groove material and the Poisson's ratio. The values of $K$ and $\mu$ are relevant to the auxiliary angle $\cos \tau$, and $\Sigma \rho$ refers to the sum of the principal curvatures of the point contact. Equation (7) could be simplified as follows:

$\delta=C \cdot Q^{2 / 3}$

$C$ is called the elastic deformation coefficient between the rolling ball and the groove.

$C=\frac{2 K}{\pi \mu} \sqrt[3]{\frac{1}{8}\left[\frac{3}{2}\left(\frac{1-v_{1}^{2}}{E_{1}}+\frac{1-v_{2}^{2}}{E_{2}}\right)\right]^{2} \sum \rho}$

For the rolling guide, the four principal curvatures of the point contact between the rolling ball and the groove are:

$\rho_{11}=\rho_{12}=\frac{2}{d_{0}}, \rho_{21}=-\frac{1}{f d_{0}}, \rho_{22}=0$

where $f$ refers to the curvature ratio, the ratio of the groove curvature radius and the diameter of the rolling ball, from which the value of $\sum \rho=\rho_{11}+\rho_{12}+\rho_{21}+\rho_{22}$ could be obtained.

In Equation (7), the values of $K$ and $\mu$ are relevant to $\cos \tau$, and in terms of the contact between the rolling ball and the groove, there is an equation as follows:

$\cos \tau=\frac{\left|\rho_{11}-\rho_{12}+\rho_{21}-\rho_{22}\right|}{\sum \rho}$

If the value of $\cos \tau$ is known, the values of $K$ and $\mu$ could be referenced from a corresponding table.

Since the material property of the carriage and the guide is the same, so is that of the groove configuration. It is considered that $C_{c}=C_{r}$. When Equations (6) and (8) are combined:

$Q_{i j}=\left(\frac{s_{i}-m_{0}}{2 C}\right)^{2 / 3}$

\subsection{The Force Balance Equation of the Slide}

Balanced forces were applied to the slide in the vertical direction, which could be expressed in the balance equation:

$\sum_{j=1}^{n}\left\{Q_{1 j} \sin \alpha_{1 j}+Q_{2 j} \sin \alpha_{2 j}\right.$
$\left.-Q_{3 j} \sin \alpha_{3 j}-Q_{4 j} \sin \alpha_{4 j}\right\}=F_{V}$

where $n$ refers to the number of rolling balls in one groove. For a guide receiving equal loads from four directions, the configuration is symmetric to the y-axis. Therefore: 
$Q_{1 j}=Q_{2 j}, Q_{3 j}=Q_{4 j}$

When Equation (11) was substituted into Equation (10):

$2 \sum_{j=1}^{n}\left\{Q_{1 j} \sin \alpha_{1 j}-Q_{3 j} \sin \alpha_{3 j}\right\}=F_{V}$

Thus, the static stiffness of the rolling guide surface could be expressed as:

$K_{V}=\frac{d F_{V}}{d v}=\frac{d}{d v}\left\{2 \sum_{j=1}^{n}\left(Q_{1 j} \sin \alpha_{1 j}-Q_{3 j} \sin \alpha_{3 j}\right)\right\}$

At this time Equations (4), (5), (9), and (12) could combine and form underdetermined nonlinear equations that included 7 equations and 11 unknown variables, which means that the number of unknowns in the model is larger than the number of equations, and that the equations are unsolvable. Therefore, the finite element analysis was needed and combined with the fitting method to solve the remaining 4 equations.

\section{THE CALCULATION METHOD FOR STIFFNESS THAT COMBINED THE FINITE ELEMENT METHOD}

\subsection{The Finite Element Model of the Carriage}

Accurate measurements of the deformation are required to learn the relationship between the carriage skirt deformation and the contact force. In this paper, the finite element method implemented in the ABAQUS software was utilized to calculate the deformation of carriage skirt.

Since the deformation only occurs at the $y-z$ and the carriages are symmetric in the y-axis, we were only required to build an axisymmetric model of the cross sections of the carriages, which is shown in Fig. (3).

During the finite element analysis, if a concentrated load is applied to a point, the local stress would be too large and leads to convergence difficulties. Therefore, there is a need to establish the functional region of the contact force based on actual conditions. According to the Hertz's theory, the area of the point contact is elliptical, and the force of the model can be expressed as:

$q_{i c}=\frac{1}{a_{i c} L_{1}} \sum_{j=1}^{n} Q_{i j}$

where $L$ refers to the length of the carriage, $a_{i c}$ refers to the major semi-axis of the contact ellipse that can be calculated through known parameters:

$a_{i c}=\sqrt[3]{\frac{6 N\left(1-v^{2}\right)}{\pi r^{2} E \sum \rho} Q_{i j}}$

If there is no restriction to the model under external forces, the analysis would be invalid. Since the model is axisymmetric in configuration, boundary conditions must be applied to the symmetric boundary. Meanwhile, a small portion of the central region on the surface of the carriage is restricted to six degrees of freedom, as shown in Fig. (3).

\subsection{The Calculation Method for Stiffness}

In this method, the non-linear equations were solved using the Levenberg-Marquardt method, which is a modification of the Gauss-Newton technique, and it is more efficient in solving non-linear equations than the GaussNewton technique.

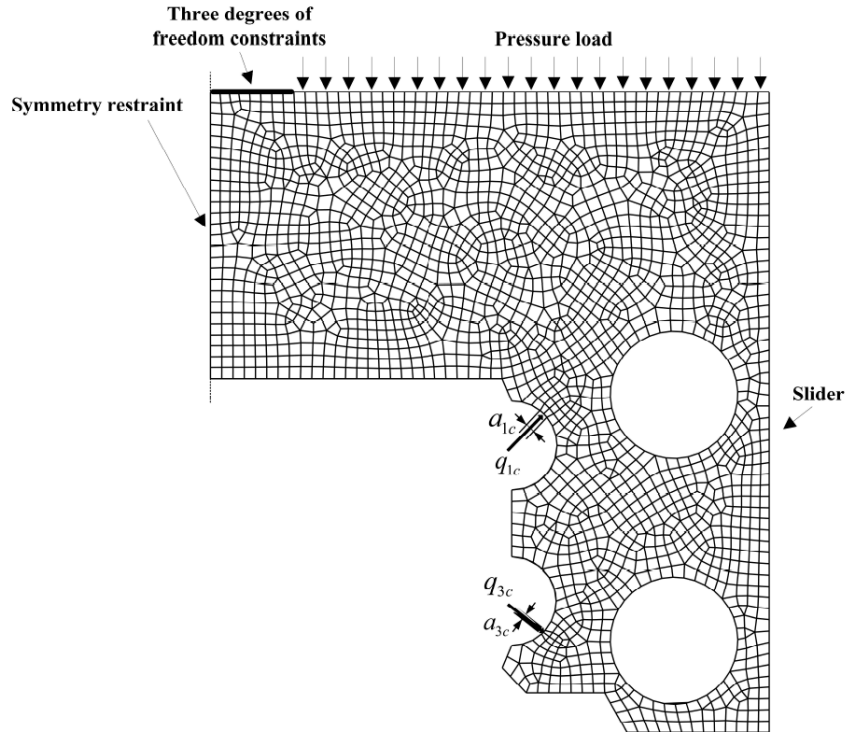

Fig. (3). The finite model of the cross section of the carriage.

The calculation process is as follows:

(1) Known variables: the curvature radius of the guide groove and carriage groove $r_{c}$ and $r_{r}$, the diameter of the rolling ball $d_{0}$, the deflection $\delta_{0}$ of the rolling ball, the initial contact angle $\alpha_{0}$ between the rolling ball and groove, the number of rolling ball $n$ that is under the force in one groove, the Young's modulus $E$, the density $\rho$ of the material of the groove, and the Poisson's ratio $v$, etc. Variables to be computed: the numerical distance between curvature center at carriage groove and center at guide groove $s_{0}$; the actual distance $m_{0}$; and the elastic coefficient $C$ for the relative deformation and contact force.

(2) A vertical force $F_{V}$ was given.

(3) The contact angle and contact force (without considering the deformation of the carriage skirt) was calculated using Hertz's theory. The non-linear equations were solved using the LevenbergMarquardt technique. At this time, the equations were given, from which the major axis of contact ellipse as well as the force of the contact area could be obtained that would be used as the boundary condition and initial load for finite element analysis.

(4) The functional area of the contact force and boundary conditions were set in the finite element software, and that the deformation $y_{c i}$ and $z_{c i}$ at all directions of the carriage groove could be obtained using static analysis under the load.

(5) The deformation values were substituted back to Equation (4), and the contact angle $\cos \alpha_{i}$ and contact force $Q_{i j}$ under this deformation were calculated using Hertz's theory. Steps (3) to (5) were repeated until at 
least three sets of values of the deformation and contact force were obtained.

(6) Polynomial interpolation fitting was used to obtain 4 equations for the deformation and contact force at all directions, i.e., $\left(y_{c l}, y_{c 3}, z_{c l}, z_{c 3}\right)=f\left(Q_{l j}\right)$.

The 4 deformation equations obtained through the Equations (4), (5), (9), and (12), and step (6) were solved using the Levenberg-Marquardt method, from which the vertical deformation under the specific vertical force was obtained.

Different vertical forces were selected when step 2 to step 7 were repeated, from which the relation between vertical deformation and the force of the groove was obtained.

(9) Based on the vertical deflection-force relation obtained in step 8, the vertical static stiffness was calculated using least squares method.

\section{METHODOLOGY AND RESULTS}

\subsection{Experimental Setup for the Guide}

In this work, a kind of guide rail with equal loads from four directions was used that was of relatively high stiffness and good performance, which was mostly used in all kinds of industrial machines and machine tools. This guide was made up of groove, carriage, block, rolling balls, and a cover plate. The rolling ball rolls on the 4 rolls made of the groove and carriage. Each roll of ball was in circular motions using the block and the cover plate. There are three levels of preloads for the guide rail, which are zero preload, light preload, and medium preload. Table 1 shows different kinds of parameters for the rail.

Table 1. Parameters for the guide rail.

\begin{tabular}{|l|c|}
\hline Elastic modulus E (Gpa) & 206 \\
\hline Poisson's ratio v & 0.3 \\
\hline Diameter of the rolling ball $(\mathrm{mm})$ & 7.938 \\
\hline Density of the material $\rho\left(\mathrm{kg} / \mathrm{m}^{3}\right)$ & 3600 \\
\hline The number of rolling balls in each groove & 45 \\
\hline Initial contact angle $\alpha_{0}(\mathrm{deg})$ & 131 \\
\hline Length of the carriage $\mathrm{L}(\mathrm{mm})$ & 100 \\
\hline Width of the carriage (mm) & 4 \\
\hline Number of grooves & 15 \\
\hline The number of rolling balls $\mathrm{n}$ under loads in each groove & 4.4 (light preload) \\
\hline \multirow{2}{*}{ Deflection of the rolling balls $(\mu \mathrm{m})$} & 7 (medium preload) \\
\hline
\end{tabular}

\subsection{Measurement of the Vertical Stiffness}

Fig. (4) shows the experimental apparatus used for measuring the vertical stiffness. Gantry side and top frames were fixed on the base using bolts. A set of linear rolling guide was fixed on the base using bolts, and corresponding carriages were fixed on the carriage. The load bolt and pressure sensor were set between the gantry top and the carriage. The displacement sensor for eddy current was fixed on the base using a magnetic stand, and its electric probe was in contact with the top surface of the carriage. The gantry framework applied vertical loads to the carriage using the load bolt. The displacement sensor was used to measure the deformation deflection of the carriage. If the deflection were measured directly, errors would occur as a result of the carriage skirt's outward expansion, making the experimental results larger.

During the loading process, the main body of the sensor was fixed using a torque wrench, and the bolt at the end of the sensor was rotated in one direction using another wrench, realizing the loading on the carriage in an easy and convenient way. The loading force ranges from 0 to $5 \mathrm{KN}$, and values were recorded using the sensor at every $1 \mathrm{KN}$ loaded, and averaged. Then, the relation between the load and the deformation deflection was obtained.

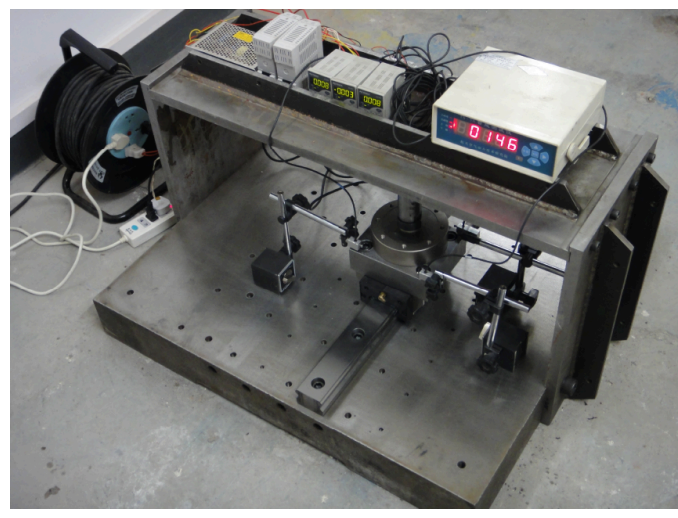

Fig. (4). Illustration of the experimental setup.

\subsection{Experimental Results}

In Fig. (5), the solid lines represent the measured results for stiffness, whereas the dotted lines represent the numerical results for stiffness obtained without considering the elastic deformation of the carriage skirt. As shown in Fig. (5), the load and the vertical deflection generally reach a linear relationship within the loading range, and the vertical stiffness under medium preload is larger than that under light preload. Under the same load, the measured vertical deflection values are larger than the numerical values, which did not consider the deformation.

Table 2. Comparison between the experimental and numerical results (not considering the deformation).

\begin{tabular}{|c|c|c|}
\hline Levels of Preload & $\begin{array}{c}\text { Light } \\
\text { Preload }\end{array}$ & $\begin{array}{l}\text { Medium } \\
\text { Preload }\end{array}$ \\
\hline Experimental results $(\mathrm{N} / \mu \mathrm{m})$ & 662.5 & 856.6 \\
\hline $\begin{array}{l}\text { Numerical results for stiffness when skirt } \\
\text { deformation was considered }(\mathrm{N} / \mu \mathrm{m})\end{array}$ & 849.6 & 1110.0 \\
\hline Relative errors (\%) & 28.2 & 29.6 \\
\hline
\end{tabular}

Table 2 shows the comparison of stiffness between the experimental and numerical results, and the measured values are $20 \%$ less. The relative errors between the numerical and experimental values almost reach $30 \%$. 


\section{THEORETICAL CALCULATION AND EXPERI- MENTAL RESULTS}

\subsection{Comparison Between Vertical Stiffness Results}

In Fig. (5), the numerical results for stiffness when the elastic deformation of the carriage skirt was considered, were shown in dash lines. As shown in Table 3, the numerical results for stiffness were closer to the experimental results when the elastic deformation of the carriage skirt was considered, with relative errors less than $6.5 \%$.

Table 3. Comparison between experimental and numerical results (skirt deformation considered).

\begin{tabular}{|c|c|c|}
\hline Levels of Preload & Light Preload & Medium Preload \\
\hline Experimental results $(\mathrm{N} / \mu \mathrm{m})$ & 662.5 & 856.6 \\
\hline $\begin{array}{l}\text { Numerical results for stiffness } \\
\text { when skirt deformation was } \\
\text { considered }(\mathrm{N} / \mu \mathrm{m})\end{array}$ & 679.9 & 912.4 \\
\hline Relative errors (\%) & 2.6 & 6.5 \\
\hline
\end{tabular}

(a) Light preload

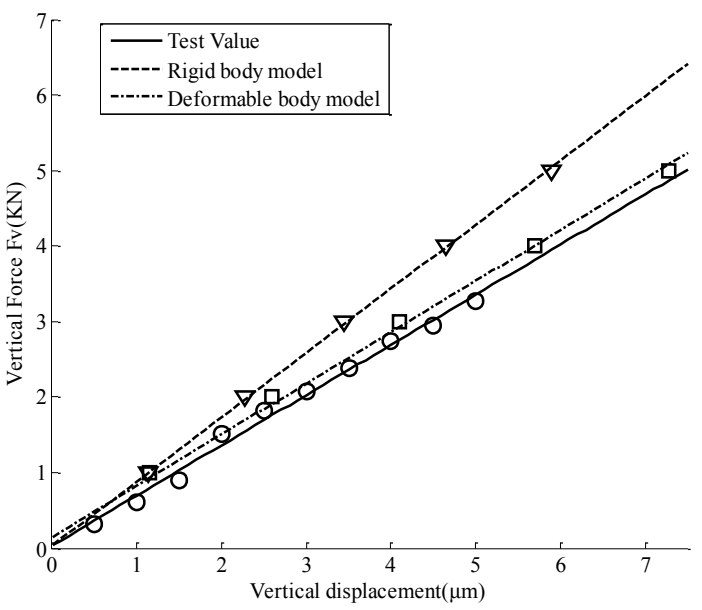

(b) Medium preload

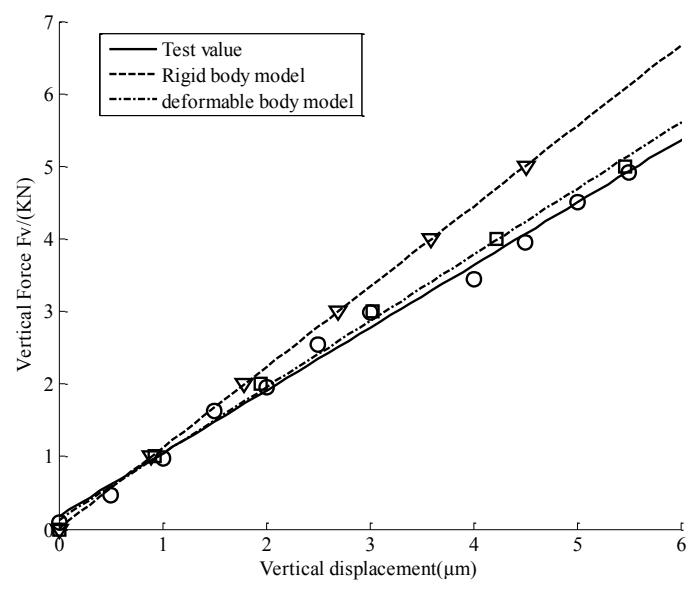

Fig. (5). Comparison between vertical stiffness values.

\subsection{Carriage Skirt Deformation}

Fig. (6) shows the carriage deformation under $1 \mathrm{KN}$ and $3 \mathrm{KN}$ preload. Under either a preload or a vertical load, the carriage deformed outward, and the deformations were smaller at the top, with increased tendency toward the bottom of the carriage. In order to make clear comparison between the deformations under different preloads and to further understand the rules of deformation, the relationship between point contact deformation and the applied load was computed when the load was raised from 0 to $5 \mathrm{KN}$, as shown in Fig. (7). (a) Light preload $1 \mathrm{KN}$

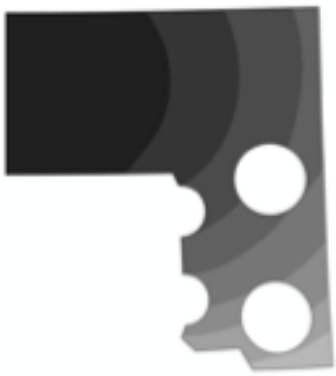

(c) Medium preload $1 \mathrm{KN}$

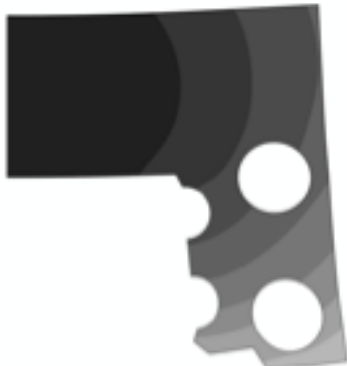

(b) Light preload $3 \mathrm{KN}$

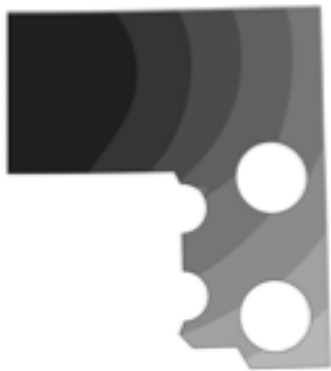

(d) Medium preload $3 \mathrm{KN}$

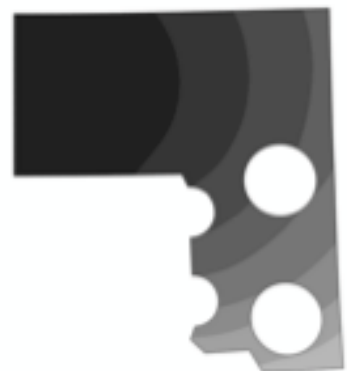

Fig. (6). Clouds of deformation of the carriage.

The numerical results for deflection show that $y_{c l}, y_{c 3}, z_{c l}$, and $z_{c 3}$ increased when the preload was raised, and exhibited almost linear relationship with the vertical load. The point contact deformation was smaller under low preload compared to that under medium preload. Under the same load, $y_{c 3}$ had the largest deformation at the bottom groove, which decreased with increased load. The other three variables $\left(y_{c l}, z_{c l}\right.$, and $\left.z_{c 3}\right)$ all increased with an increased load. $y_{c l}$ and $z_{c l}$ exhibited small variations when the load changed, and the curve becomes more linear. On the other hand, $y_{c 3}$ and $z_{c 3}$ exhibited greater variations with an increased load. Therefore, skirt deformation is a result of the combined effect of preload and vertical load, both of which should be considered in such studies in order to calculate more accurate results for static stiffness.

\section{CONCLUSION}

In this paper, a theoretical model for vertical stiffness was established taking into account the deformation of carriage skirt, and the corresponding calculation method was reported;

(2) The numerical results of stiffness that took into account the skirt deformation, were closer to the measured values compared to those that ignored the deformation, with relative errors no larger than $6.5 \%$; 
(a) Light preload

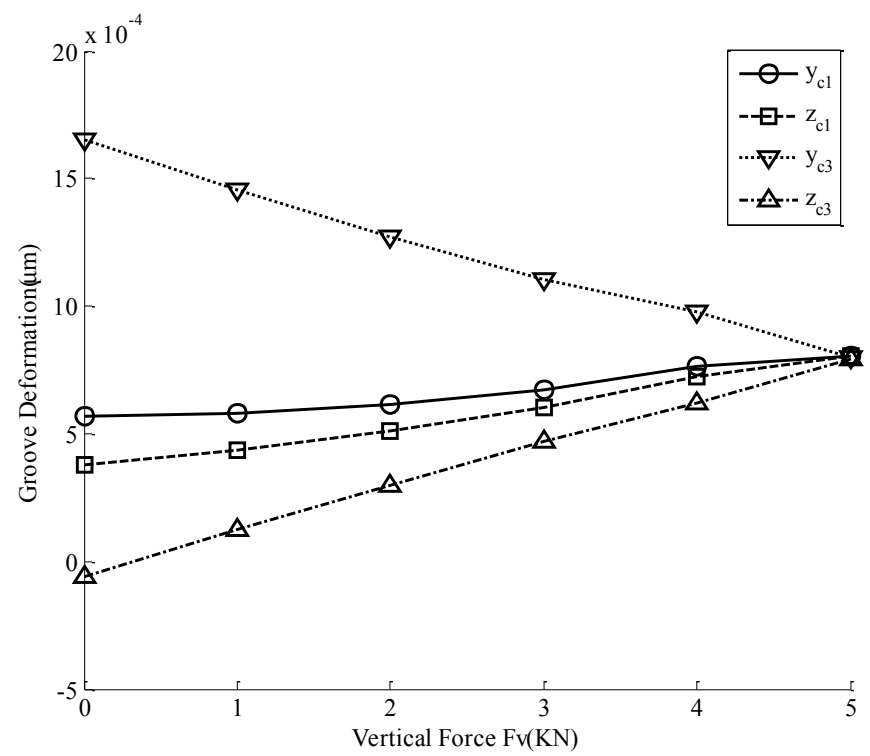

(b) Medium preload

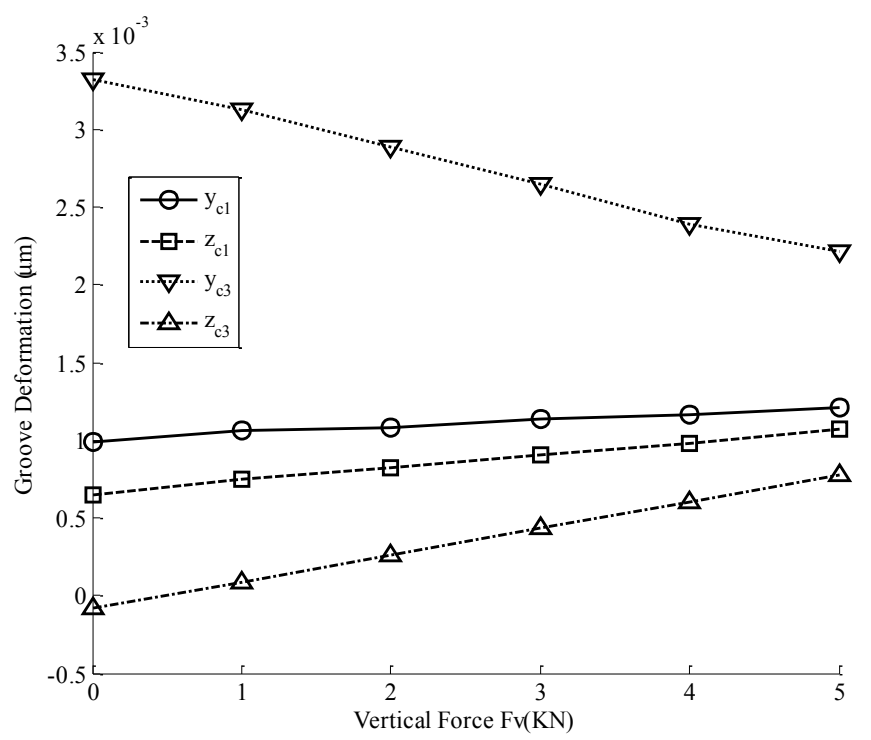

(3) Under the preload and vertical load of the carriage, the skirt exhibited a tendency to deform outwardly, which increased from the top to the bottom. Therefore, only when the influence of both the preload and the vertical load on deformation is considered, can the static stiffness be estimated more accurately.

\section{CONFLICT OF INTEREST}

The authors confirm that this article content has no conflict of interest.

\section{ACKNOWLEDGEMENTS}

It is a project supported by the National Science Fund for Distinguished Young Scholars (51225503).

\section{REFERENCES}

[1] J. L. Sun, "Stiffness calculation under vertical load for linear rolling guide system," Journal of Huazhong University of Science and Technology, vol. 16, no. 5, pp. 19-24, 1988.

[2] J. L. Sun, "The preloading and stiffness calculation for precise linear rolling guide," Journal of Huazhong University of Science and Technology, vol. 16, no. 6, pp. 125-130, 1988.

[3] S. Shimizu, "Load distribution and accuracy/rigidity of linear motion ball guide system," Journal of the Japan Society of Precision Engineering, vol. 56, no. 8, pp. 1445-1451, 1990.

[4] D. Liu, J.L. Sun, and D.X. Liao, "Stiffness calculation considering the carriage elastic deformation of the linear rolling guide," Journal of Huazhong University of Science and Technology, vol. 18, no. 8, pp. 235-240, 1990.

[5] T. Chen, and Q.B. Huang, "A vertical stiffness model that involves the carriage skirt deformation of the rolling linear guide," China Mechanical Engineering, vol. 22, no. 13, pp. 1546-1550, 2011.

[6] H. Ohta, and K. Tanaka, "Vertical stiffnesses of preloaded linear guide type ball bearings incorporating the flexibility of the carriage and groove," ASME Journal of Tribology, vol. 129, pp. 188-193, 2010 .

Fig. (7). Illustration of the relation between groove deformation and load. 